\title{
Das verspätete Aufräumen
}

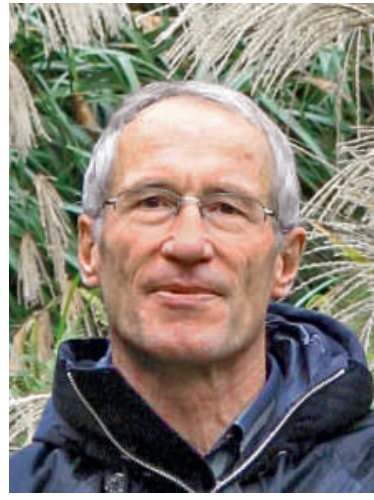

Erhard Taverna
Gemäss der Frankfurter Allgemeinen Zeitung übergab die Charité Ende September 2011 zwanzig Schädel aus ihrer medizinhistorischen Sammlung an eine Delegation aus Namibia. Es sei dies eine Geste des Respekts und des Bedauerns, hiess es aus der berühmten Berliner Klinik, mit der man zum ehrenvollen Andenken der Toten beitragen wolle. Um diese Zeremonie im Beisein der Staatsministerin im Auswärtigen Amt zu verstehen, muss man in die Geschichte Deutschlands zurückblenden. Die Schädel gehörten Opfern von Stammesangehörigen der Herero und Nama, die sich im ehemaligen Deutsch-Südwestafrika 1904 gegen die Kolonialherrschaft erhoben hatten. Die blutige Niederschlagung des Aufstands und die folgenden, systematischen Massaker gelten als erster Völkermord des letzten Jahrhunderts. Zehntausende Kämpfer und ihre Familien wurden damals in die Wüste getrieben, wo sie qualvoll umkamen. Die präparierten Schädel vieler Toter wurden an deutsche Universitäten $\mathrm{zu}$ «rassenanatomischen Untersuchungen» geschickt. Seit 2010 arbeitet die Charité an der Geschichte ihrer Sammlung. Die auf zwei Jahre angelegte Studie «Human Remains Project» hat das Ziel, Herkunft und Erwerb der grossen anthropologischen Schädel- und Skelettsammlungen zu untersuchen sowie Kriterien für Rückgabeverhandlungen zu erarbeiten.

\section{«Alle Kolonialstaaten hatten oder haben ähnliche Leichen im Keller.»}

Alle Kolonialstaaten hatten oder haben ähnliche Leichen im Keller. 1976 veranlasste die Regierung Tasmaniens, unter dem Druck der schwarzen und weissen Bevölkerung, die Feuerbestattung eines Skeletts, das im Museum der Royal Society in Hobart ausgestellt war. Die 1876 verstorbene Ureinwohnerin war die letzte ihres ausgerotteten Volkes. Zwei Jahre nach der Beerdigung wurde sie für «wissenschaftliche Zwecke» exhumiert. Ansprüche auf Landrückgabe oder Entschädigung an die Nachfahren waren damit allerdings nicht verbunden.

Ein Senatsprojekt der Universität Wien begann 1998, die Herkunft der Leichen des Anatomischen Instituts von 1938-1945 zu untersuchen. Amerikanische, kanadische und israelische Mediziner hatten seit den 1980er Jahren eine Überprüfung der Präparate gefordert und nach der Entstehungsgeschichte des bekann- ten Anatomieatlasses von Eduard Pernkopf gefragt. Dieser war zunächst Dekan und danach Rektor der Universität bis 1945. Nach der Zwangspensionierung und drei Jahren Haft in einem Lager der Alliierten, setzte er, wie die meisten seiner Medizinerkollegen, ungehindert seine Arbeit fort. Über 1300 Leichen von Opfern der NS-Justiz, darunter acht Personen jüdischer Herkunft, waren im fraglichen Zeitraum dem Institut zur wissenschaftlichen Forschung und für den Unterricht übergeben worden. Pernkopfs «Topografische Anatomie des Menschen, Atlas der regionär-stratigrafischen Präparation» erschien von 1937 bis 1960 und gilt bis heute als Standardwerk. Histologische Schnittpräparate wurden an allen führenden Universitäten Österreichs und Deutschlands bis in die 1990er Jahre für den Unterricht der Medizinstudenten verwendet. Viele davon, vor allem die der Neurologie, stammten aus dem berüchtigten T4-EuthanasieProgramm, dem viele Psychiatriepatienten und körperlich Behinderte zum Opfer fielen. Die meisten anatomischen Institute besassen ihre eigenen Schädel- und Skelettsammlungen, die den pseudo-wissenschaftlichen Untersuchungen für die ideologisch vorgegebenen «rassenkundlichen»Ziele dienten. An einem Massengrab im jüdischen Friedhof von Strassburg wurde 2005 ein Grabstein mit 86 Namen aufgrund ähnlicher Recherchen errichtet. Alle ermordeten Häftlinge hatte der Institutsleiter schweizerischer Abstammung, Professor August Hirt, persönlich im KZ ausgesucht und für seine vorgesehene Sammlung ermorden lassen.

Seit Vesalius' Werk «De Humani Corporis fabrica» sind Hingerichtete für anatomische Sektionen verwendet worden. Ein in den USA zum Tode verurteilter Mann diente als Vorlage für einen neuartigen CT-Atlas, ein Teil von Hagens' Plastinaten soll von Exekutierten aus China stammen. Möglicherweise werden eines Tages ihre Nachfahren die Herausgabe dieser sterblichen Relikte verlangen. Wo sich politischer Druck aufbaut, müssen sich in Zukunft ethnologische und anthropologische Sammlungen von gewohnten Exponaten trennen. Seit dem Nürnberger Kodex regeln zahlreiche Abkommen die ethischen Voraussetzungen für korrekte Humanversuche. Seit der Annahme des eidgenössischen Humanforschungsgesetzes im März 2010 besteht ein präventives Regelwerk. Damit bleibt zu hoffen, dass auch nach weniger friedlichen Zeiten keine medizinische Fakultät jemals in die Lage kommt, in den anatomischen Sammlungen aufräumen zu müssen.

Erhard Taverna 